\title{
¿Cómo son derivadas las pacientes con cáncer de mama a la consulta de oncología médica?
}

\section{Sr. Director:}

En la actualidad el adecuado desarrollo del Hospital de Día de Oncología y de las consultas externas, supone asegurar una asistencia de calidad, pues ambas representan más del $80 \%$ de la carga asistencial que tiene el médico dedicado a la oncología.

La obtención de diversos parámetros de medida de la actividad asistencial en éstas áreas sirve de gran ayuda y debería constituir una práctica prioritaria para mejorar los resultados asistenciales y realizar una adecuada previsión de los recursos.

Presentamos los resultados de un pequeño estudio en el que analizábamos diversos parámetros de las pacientes con cáncer de mama, intervenidas por $\mathrm{Ci}$ rugía General y Obstetricia y Ginecología y posteriormente derivadas a las consultas de oncología médica de un hospital del grupo 2.

El estudio realizado era observacional prospectivo de prevalencia, analizando cinco parámetros básicos en las pacientes derivadas a la consulta de Oncología y que eran : 1) Si constaba el resultado definitivo de la histología tras la cirugía en el informe de alta y utilizado para la derivación a las consultas. 2) Si se había realizado un estudio de extensión previo a la intervención quirúrgica, consistente en radiografía de tórax, gammagrafía o serie ósea y ecografía hepática. 3) Si se especificaba el estadio del tumor siguiendo la clasificación TNM. 4) Si se había efectuado una cirugía adecuada en su extensión al estadio de la enfermedad y características de la enferma. 5) Si la derivación se había realizado siguiendo un perfil temporal adecuado, admitiendo como tal desde los 15 hasta los 30 días tras la cirugía; siempre que no hubiese presentado complicaciones postoperatorias que obligasen a una mayor demora.
En el periodo del estudio analizamos 44 derivaciones de pacientes intervenidas de cáncer de mama, con edades comprendidas entre 35 y 87 años con una edad media de 58,2 años.

Cumplían la totalidad de los 5 parámetros analizados, únicamente 4 pacientes $(9 \%)$.

Revisando por separado cada parámetro, cumplían el primero (constaba la histología de la pieza quirúrgica) en 41 de las 44 enfermas (93\%).

Tenían realizado un estudio de extensión previo a la cirugía, 25 de las 44 enfermas $(56,8 \%)$.

Constaba el estadio en el informe de alta o de derivación en 8 de las 44 enfermas (18,9\%).

La cirugía realizada era indicada a la extensión de la enfermedad en 41 de 44 enfermas (93\%). En otras dos, los márgenes se encontraban afectados y sin embargo se derivó a la consulta de oncología sin haber ampliado estos márgenes antes y en otra enferma se había efectuado una mastectomía sin realizar previamente el estudio de extensión y en la gammagrafía ósea se detectó infiltración ósea múltiple, por lo que la mastectomía no había estado indicada.

La derivación se había realizado con un perfil temporal adecuado (entre 15 y 30 días) en 41 de las 44 enfermas (93\%). En otras dos, se realizó después de completar la radioterapia, lo que motivo una demora de dos meses y medio y en la otra enferma se realizó tras una revisión en cirugía, pasados ya tres meses de la intervención quirúrgica.

Pese a su importancia, nos llama la atención la nula bibliografía que hemos encontrado sobre esta problemática, pues si bien hay numerosos trabajos de organización y de gestión, ninguno trata de este tema específicamente.

Como conclusiones de nuestro pequeño estudio, 
lo que más nos llamó la atención es el escaso porcentaje de pacientes a las que se realizó el estudio de extensión previo a la cirugía, únicamente un 56,8\%, cuando debería ser el $100 \%$.

El porcentaje mínimo de pacientes que tenían indicado el estadio (18,9\%), es muy problema menos relevante, pues ya lo realizábamos nosotros con posterioridad y esto no redundaba en ningún perjuicio para las pacientes.

Los otros 3 parámetros estaban por encima del $90 \%$, pero pensamos que deberían ser el $100 \%$, por lo que es necesario mejorar también en este sentido.

\section{F. Marcos Sánchez, $M^{\mathrm{a}}$ I. Albo Castaño, A. Viana Alonso, S. Casallo Blanco, F. Juárez Ucelay Servicio de Medicina Interna. Hospital $N^{a} S^{a}$ del Prado. Talavera de la Reina. Toledo.}

Correspondencia:

Dr. F. Marcos Sánchez

C/ Gregorio Corrochano, 1

E-45600 Talavera de la Reina (Toledo) 